\title{
Perioperative D-dimer levels after transcatheter aortic valve replacement: Comparison of patients with and without anticoagulant therapy
}

\author{
Akihiro Tobe ${ }^{1}$, Akihito Tanaka ${ }^{1} \oplus$, Yoshiyuki Tokuda ${ }^{2}$, Toshihiko Nishi ${ }^{2}$, Yusuke Miki ${ }^{1}$, \\ Kenji Furusawa ${ }^{1}$, Hideki Ishii ${ }^{1,3}$, Akihiko Usui ${ }^{2}$, Toyoaki Murohara ${ }^{1}$ \\ ${ }^{1}$ Department of Cardiology, Nagoya University Graduate School of Medicine, Nagoya, Japan \\ ${ }^{2}$ Department of Cardiac Surgery, Nagoya University Graduate School of Medicine, Nagoya, Japan \\ ${ }^{3}$ Department of Cardiology, Fujita Health University Bantane Hospital, Nagoya, Japan
}

Transcatheter aortic valve replacement (TAVR) has become an established treatment option for severe aortic valve stenosis. Given this situation, leaflet thrombosis of prosthesis after TAVR has been raised as a persisting concern, as it might cause systemic embolism or valve failure [1]. A prior report showed the relationship between leaflet thrombosis on enhanced computed tomography and elevated D-dimer level [2], which is a product of fibrinolysis and is used as a sensitive marker of intravascular thrombus [3]. An antiplatelet agent is used in the current standard antithrombotic regimen after TAVR; however, some recent reports suggest that patients treated with anticoagulant therapy after TAVR were at lower risk of leaflet thrombosis than those treated without anticoagulant therapy $[4,5]$. The purpose of this study was to compare the D-dimer levels during the perioperative period of TAVR between patients with and without anticoagulant therapy.

From among 114 consecutive patients who received TAVR between April 2016 and June 2019 at Nagoya University Hospital, subjects were identified with available perioperative D-dimer levels (more than once within 7 days after TAVR). Patients were excluded if their postoperative $\mathrm{D}$-dimer levels were not available $(n=19)$, anticoagulant therapy was changed to antiplatelet therapy or vice versa within 7 days $(n=2)$, antithrombotic therapy was not started within 5 days of TAVR $(n=5)$, or the baseline $\mathrm{D}$-dimer level before TAVR was extremely high (D-dimer level > $10 \mathrm{IU} / \mathrm{mL} ; \mathrm{n}=1$ ).
Finally, 87 patients were analyzed in this study. The highest D-dimer levels were defined within 7 days after TAVR as "peak D-dimer" and the D-dimer level before TAVR as "baseline D-dimer". $\Delta \mathrm{D}$-dimer was also calculated with peak and baseline D-dimer levels.

Categorical variables are presented as numbers and percentages, and continuous variables are presented as mean \pm standard deviation or median (interquartile range). To compare the categorical variables, $\chi^{2}$ or the Fisher exact test was used. Continuous variables were compared using a t test or the Mann-Whitney test. $\mathrm{P}$ values $<0.05$ were considered to indicate statistical significance. All statistical analyses were performed using SPSS version 26.0 (SPSS, Chicago, Illinois).

Patient characteristics are shown in Table 1. Of the 87 patients, 21 received anticoagulants (2 received warfarin; 12 , direct oral anticoagulants [DOAC]; and 7, both DOAC and single antiplatelet agents). Seventeen patients received anticoagulants for atrial fibrillation at baseline, and the other 4 patients started taking anticoagulants after TAVR. Sixty-six patients received only antiplatelet agents without anticoagulants (11 received dual and 55 received single antiplatelet therapy). The patients treated with anticoagulant therapy had atrial fibrillation more frequently.

The perioperative $\mathrm{D}$-dimer levels are shown in Table 1 . The peak D-dimer level was significantly lower in the patients treated with anticoagulants than in those treated without anticoagulants (2.38

Address for correspondence: Dr. Akihito Tanaka, MD, PhD, Department of Cardiology, Nagoya University Graduate School of Medicine, 65 Tsurumai-cho, Showa-ku, Nagoya 466-8560, Japan, tel: +81-52-741-2111, fax: +81-52-744-2138,

e-mail: akihito17491194@gmail.com

Received: 3.04 .2020

This article is available in open access under Creative Common Attribution-Non-Commercial-No Derivatives 4.0 International (CC BY-NC-ND 4.0) license, allowing to download articles and share them with others as long as they credit the authors and the publisher, but without permission to change them in any way or use them commercially. 
Table 1. Baseline characteristics and perioperative D-dimer levels.

\begin{tabular}{|c|c|c|c|}
\hline & $\begin{array}{l}\text { Anticoagulant } \\
\quad(n=21)\end{array}$ & $\begin{array}{c}\text { Non-anticoagulant } \\
(\mathrm{n}=66)\end{array}$ & $\mathbf{P}$ \\
\hline \multicolumn{4}{|l|}{ Baseline characteristics } \\
\hline Age [years] & $84 \pm 4$ & $84 \pm 5$ & 0.51 \\
\hline Male & $7(33 \%)$ & $22(33 \%)$ & 1.00 \\
\hline Height $[\mathrm{cm}]$ & $148(146-160)$ & $147(143-153)$ & 0.20 \\
\hline Body weight [kg] & $48(42-54)$ & $48(41-61)$ & 0.68 \\
\hline Body mass index & $21.8(19.2-26.6)$ & $22.5(18.9-26.7)$ & 0.17 \\
\hline Hypertension & $16(76 \%)$ & $50(76 \%)$ & 0.97 \\
\hline Dyslipidemia & $14(67 \%)$ & $38(58 \%)$ & 0.46 \\
\hline Diabetes mellitus & $7(33 \%)$ & $23(35 \%)$ & 0.90 \\
\hline eGFR $<60 \mathrm{~mL} / \mathrm{min} / 1.73 \mathrm{~m}^{2}$ & $12(57 \%)$ & $48(73 \%)$ & 0.18 \\
\hline Atrial fibrillation & $17(81 \%)$ & $2(3 \%)$ & $<0.001$ \\
\hline Extracardiac arteriopathy & $4(19 \%)$ & $9(14 \%)$ & 0.38 \\
\hline Active cancer & $2(10 \%)$ & $0(0 \%)$ & 0.06 \\
\hline Previous cardiac surgery & $3(15 \%)$ & $4(6 \%)$ & 0.22 \\
\hline STS score & $5.9(5.0-8.9)$ & $5.6(4.4-7.6)$ & 0.41 \\
\hline \multicolumn{4}{|l|}{ Echocardiography } \\
\hline Left ventricular ejection fraction [\%] & $66.5(62.0-75.0)$ & $64.5(59.0-71.9)$ & 0.34 \\
\hline Mean pressure gradient [mmHg] & $43.3(38.7-51.2)$ & $43.5(36.0-62.1)$ & 0.78 \\
\hline Aortic valve area $\left[\mathrm{cm}^{2}\right]$ & $0.57 \pm 0.15$ & $0.60 \pm 0.18$ & 0.38 \\
\hline \multicolumn{4}{|l|}{ Computed tomography } \\
\hline Annulus area $\left[\mathrm{cm}^{2}\right]$ & $420.9 \pm 63.9$ & $410.7 \pm 76.8$ & 0.59 \\
\hline Annulus perimeter $[\mathrm{mm}]$ & $73.9 \pm 5.6$ & $73.0 \pm 6.2$ & 0.62 \\
\hline Mean sinus of Valsalva diameter [mm] & $29.6 \pm 3.1$ & $29.5 \pm 3.1$ & 0.95 \\
\hline \multicolumn{4}{|l|}{ Procedural characteristics } \\
\hline Approach site: & & & 0.55 \\
\hline Transfemoral & $20(95 \%)$ & $61(92 \%)$ & \\
\hline Non-transfemoral & $1(5 \%)$ & $5(8 \%)$ & \\
\hline Prosthesis type: & & & 0.79 \\
\hline SAPIEN XT/3 & $14(67 \%)$ & $46(70 \%)$ & \\
\hline Evolut R/Pro & $7(33 \%)$ & $20(30 \%)$ & \\
\hline \multicolumn{4}{|l|}{ Antithrombotic therapy: } \\
\hline Warfarin only & 2 & 0 & \\
\hline DOAC only & 12 & 0 & \\
\hline DOAC + SAPT & 7 & 0 & \\
\hline DAPT & 0 & 11 & \\
\hline SAPT & 0 & 55 & \\
\hline \multicolumn{4}{|l|}{ Perioperative D-dimer levels } \\
\hline Baseline D-dimer level [IU/mL] & $0.50(0.50-0.65)^{*}$ & $1.10(0.64-1.95)^{*}$ & 0.003 \\
\hline Peak D-dimer level [IU/mL] & $2.38(1.74-4.29)$ & $5.15(2.64-8.51)$ & 0.001 \\
\hline$\Delta \mathrm{D}$-dimer & $1.56(0.83-2.48)^{*}$ & $5.05(2.10-7.76)^{*}$ & $<0.001$ \\
\hline
\end{tabular}

eGFR - estimated glomerular filtration rate; STS - Society of Thoracic Surgeons; DOAC - direct oral anticoagulant; DAPT — dual antiplatelet therapy; SAPT — single antiplatelet therapy; $\triangle \mathrm{D}$-dimer - peak minus baseline D-dimer levels; *Baseline D-dimer levels were available in 16 patients with anticoagulant and 46 patients without anticoagulant, therefore, $\Delta \mathrm{D}$-dimer was calculated in those patients

$\mathrm{IU} / \mathrm{mL}[1.74-4.29 \mathrm{IU} / \mathrm{mL}]$ vs. $5.15 \mathrm{IU} / \mathrm{mL}[2.64-$ $-8.51 \mathrm{IU} / \mathrm{mL}] ; \mathrm{p}=0.001)$. The baseline $\mathrm{D}$-dimer $(0.50 \mathrm{IU} / \mathrm{mL}[0.50-0.65 \mathrm{IU} / \mathrm{mL}]$ vs. $1.10 \mathrm{IU} / \mathrm{mL}$
[0.64-1.95 IU $/ \mathrm{mL}] ; \mathrm{p}=0.003)$ and $\Delta$-dimer levels $(1.56 \mathrm{IU} / \mathrm{mL}[0.83-2.48 \mathrm{IU} / \mathrm{mL}]$ vs. $5.05 \mathrm{IU} / \mathrm{mL}$ [2.10-7.76 IU/mL]; $\mathrm{p}<0.001)$ were significantly 
lower in the patients with, than in those without anticoagulant therapy.

Preliminary data showed that the baseline, peak, and D-dimer levels were significantly lower in patients with anticoagulant therapy. Recent reports suggest the effectiveness of anticoagulant therapy for prevention and treatment of leaflet thrombosis after TAVR [4, 5], and the present data may support these results. D-dimer levels could be affected by various factors, and suppression of the increase in D-dimer level might not directly demonstrate the effectiveness of anticoagulant therapy. Prior reports have shown that D-dimer levels decreased in patients with atrial fibrillation after initiation of DOAC therapy [6], and anticoagulant therapy might suppress the coagulation reaction and formation of leaflet thrombosis of prosthesis after TAVR. However, the effectiveness of a prevention for leaflet thrombosis does not mean it is the optimal therapy for patients undergoing TAVR $[7,8]$ because the balance between safety and efficacy for the various issues should be considered in clinical practice. Further investigations are required to address the optimal antithrombotic regimen in patients undergoing TAVR.

Conflict of interest: Hideki Ishii received lecture fees from Astellas Pharma Inc., Astrazeneca Inc., Daiichi-Sankyo Pharma Inc., and MSD K.K. Akihiko Usui received lecture fees from Terumo, Japan Blood Products Organization. Akihiko Usui received unrestricted research grant for Department of Cardiac Surgery, Nagoya University Graduate School of Medicine from Edwards Lifesciences Corporation, Senko Medical Instrument Mfg., Co., Ltd., Medtronic, Terumo, and Lifline. Toyoaki Murohara received lecture fees from Bayel Pharmaceutical Co., Ltd., Daiichi-Sankyo Co., Ltd., Dainippon Sumitomo Pharma Co., Ltd., Kowa Co., Ltd., MSD K.K., Mitsubishi Tanabe Pharma Co., Nippon Boehringer Ingelheim Co., Ltd., Novartis Pharma K.K., Pfizer Japan Inc., Sanofi-Aventis K.K., and Takeda Pharmaceutical Co., Ltd. Toyoaki Murohara received unrestricted research grant for Depart- ment of Cardiology, Nagoya University Graduate School of Medicine from Astellas Pharma Inc., Daiichi-Sankyo Co., Ltd., Dainippon Sumitomo Pharma Co., Ltd., Kowa Co., Ltd., MSD K.K., Mitsubishi Tanabe Pharma Co., Nippon Boehringer Ingelheim Co., Ltd., Novartis Pharma K.K., Otsuka Pharma Ltd., Pfizer Japan Inc., Sanofi-Aventis K.K., Takeda Pharmaceutical Co., Ltd., and Teijin Pharma Ltd. All other authors have reported that they have no relationships relevant to the contents of this paper to disclose.

\section{References}

1. Jose J, Sulimov DS, El-Mawardy M, et al. Clinical bioprosthetic heart valve thrombosis after transcatheter aortic valve replacement: incidence, characteristics, and treatment outcomes. JACC Cardiovasc Interv. 2017; 10(7): 686-697, doi: 10.1016/j. jcin.2017.01.045, indexed in Pubmed: 28385406.

2. Yanagisawa R, Hayashida K, Yamada Y, et al. Incidence, predictors, and mid-term outcomes of possible leaflet thrombosis after TAVR. JACC Cardiovasc Imaging. 2016 [Epub ahead of print], doi: 10.1016/j.jcmg.2016.11.005, indexed in Pubmed: 28017712.

3. Olson JD. D-dimer: an overview of hemostasis and fibrinolysis, assays, and clinical applications. Adv Clin Chem. 2015; 69: 1-46, doi: 10.1016/bs.acc.2014.12.001, indexed in Pubmed: 25934358.

4. Chakravarty T, Søndergaard L, Friedman J, et al. Subclinical leaflet thrombosis in surgical and transcatheter bioprosthetic aortic valves: an observational study. Lancet. 2017; 389(10087): 2383-2392, doi: 10.1016/S0140-6736(17)30757-2, indexed in Pubmed: 28330690.

5. Nijenhuis VJ, Brouwer J, Søndergaard L, et al. Antithrombotic therapy in patients undergoing transcatheter aortic valve implantation. Heart. 2019; 105(10): 742-748, doi: 10.1136/ heartjnl-2018-314313, indexed in Pubmed: 30867148.

6. Nagao T, Hunakubo H, Suzuki M, et al. Trends in physiological coagulation factors in Japanese patients receiving novel oral anticoagulants. J Arrhythm. 2017; 33(2): 117-121, doi: 10.1016/j. joa.2016.07.011, indexed in Pubmed: 28416977.

7. Dangas GD, Tijssen JGP, Wöhrle J, et al. A controlled trial of rivaroxaban after transcatheter aortic-valve replacement. $\mathrm{N}$ Engl J Med. 2020; 382(2): 120-129, doi: 10.1056/NEJMoa1911425, indexed in Pubmed: 31733180.

8. De Backer O, Dangas GD, Jilaihawi H, et al. Reduced leaflet motion after transcatheter aortic-valve replacement. N Engl J Med. 2020; 382(2): 130-139, doi: 10.1056/NEJMoa1911426, indexed in Pubmed: 31733182. 